\title{
Assessment of an Open-Source Pressure-Based Real Fluid Model for Transcritical Jet Flows
}

\author{
Faniry N.Z. Rahantamialisoa*1, Adrian Pandal ${ }^{2}$, Ningegowda B. M. ${ }^{1}$, Jacopo Zembi ${ }^{1}$, Nasrin \\ Sahranavardfard ${ }^{1}$, Hrvoje Jasak ${ }^{3}$, Hong G. Im $^{4}$, Michele Battistoni ${ }^{1}$ \\ ${ }^{1}$ Department of Engineering, Università degli di Studi Perugia, Perugia, Italy \\ ${ }^{2}$ Departement of Energy, Universidad de Oviedo, Gijon, Spain \\ ${ }^{3}$ Department of Physics, University of Cambridge, Cambridge, UK \\ ${ }^{4}$ Clean Combustion Research Center, King Abdullah University of Science and Technology, \\ Thuwal, Saudi Arabia \\ *Corresponding author email: fanirynadiazazaravaka.rahantamialisoa@studenti.unipg.it
}

\begin{abstract}
Complexity behind physical phenomena of supercritical and transcritical jet flows, still leaves an ambiguous understanding of such widespread technology, with applications ranging from diesel and liquid rocket engines to gas turbines. In this present numerical study, a new open-source CFD model construction is presented and validated using a liquid-rocket benchmark comprised of liquid-oxygen (LOX) and gaseous-hydrogen (H2) streams. Mixing process of liquid oxygenhydrogen streams under liquid rocket engine (LRE) relevant conditions is scrutinized using the pressure-based solution framework implemented in the versatile computation platform OpenFOAM. The model accounts for real fluid thermodynamics and transport properties, making use of the cubic Peng-Robinson equation of state (PR-EOS) and the Chung transport model. The solver capability to capture the mixing layer between the two separated streams is discussed as well as its capability to predict with adequate accuracy the thermophysical quantities. Following the thorough validation, a comparison of the contribution of the accurate laminar transport properties vs. the large eddy simulation (LES) subgrid scale (sgs) turbulent values is conducted in order to assess the relative importance of the turbulent viscosity. By means of an assessment of the pressure-based numerical framework with available data in the literature, this work contributes to a better understanding of well resolved simulations. In addition, it enables the further development of a real fluid pressure-based multi-species solver as an open-source code.
\end{abstract}

\section{Keywords}

Supercritical flow, Real-fluid thermodynamics, LES, CFD, LOX-H2, liquid rocket engines.

\section{Introduction}

Modern high-performance propulsion and transportation devices from rocket engines to diesel engines involve injections occurring at high pressure. For liquid-fueled rocket engines, this translates to propellants injected at a super- or trans-critical state, i.e., at pressures above the critical pressure of the fluid and temperatures close to or below the critical temperature. In this context, such situations come with changes in the physical characteristics and behaviour compared to classical sub-critical injection and fluid properties are highly influenced by changes in temperature. Fluid behaviour deviates from the ideal gas as the assumption of negligible molecule volume does not hold anymore and also there is a diminution of the surface tension [1]. Besides, as the fluid undergoes the transcritical path, it crosses the pseudo-boiling line along which some thermodynamic properties (heat capacity, thermal expansion coefficient, isothermal compressibility) are at their maxima. Hence, detailed understanding of these complex operating conditions is crucial. Many theoretical and experimental studies have been conducted over the years regarding high pressures flows: from Mayer and Tamura [2] that conducted experimental visualizations of supercritical injection and mixing processes of Liquid Oxygen (LOX) and Gaseous Hydrogen (H2) to Habiballah et al. [3] and their investigations on 
the differences of flame structure in sub- and super-critical regimes with Mascotte benchmark test case. Experiments of a cold supercritical nitrogen jet that is injected into an ambient temperature nitrogen environment were also conducted by Mayer et al. [4]. Further experiments regarding inert binary injection and mixing processes were presented by Oschwald et al. [5]. Both studies underline that the prevailing physical phenomena change when the operating pressure exceeds the critical point. Even though, those works have allowed to make remarkable progress in order to gain deeper insight into such complex phenomena, still limited information can be captured through experiments. This has motivated the growing efforts towards modeling studies of trans-critical and super-critical flows. Several works have been carried out to mimic Mayer et al. experiments: like in [6] or in [7] where the role of sgs model were investigated for a jet of cryogenic nitrogen or in [8] which among their results provides an analysis of the heat transport phenomena within a three dimensional Direct Numerical Simulation (DNS). Reitz and co-workers focused on a thermodynamic analysis of the mixtures states [9]. The group of $\mathrm{J}$. Bellan $[10,11]$ has extensively studied shear layers in a supercritical environment using both (DNS) and Large Eddy Simulation (LES). Similar works have also been reported in $[12,13]$. On the other hand, numerical studies are also subjected to challenges. The difficulties arise mainly from real-gas effects that strongly influence these processes, leading to non-linearities of the thermodynamic system and thereby non-physical oscillations of the pressure which can severely impact the accuracy. It has been therefore the main focus of many researchers to develop numerical strategies in order to achieve both stability and accuracy [7,14-18].

This present study follows previous works carried out in [19] to simulate multicomponent mixture species spray mixing processes with real-fluid thermophysical properties on both cryogenic and non-cryogenic fuel injections. However, here the thermophysical models have been modified to incorporate more convenient mixing rules. It is then one of the main objective of this work to make further validations of the pressure-based framework with available data in the literature. In fact, Ruiz et al. [20] provides a comprehensive data set with a benchmark test case at highReynolds-number supercritical flow with large density gradients. The case consists of a two dimensional DNS of LOX-H2 streams mixing layer separated by an injector lip and is taken up by Ma et al. in [16] among other test cases to validate the robustness of the proposed entropy stable hybrid scheme or by Lacaze et al. [17] that reproduce the same configuration to address an extensive comparison of three numerical approaches based on different formulations of the transported properties. Furthermore, LES results are also presented in the present numerical study to characterize the influence of the filtering approach on the mixing prediction and to assess the relative importance of the turbulent viscosity vs. the real-fluid laminar one.

\section{Case Setup}

As aforementioned, the benchmark case proposed in [20] is reproduced in the present work and it is represented in Figure 1 with the boundary conditions. Under LRE relevant conditions with a pressure set at 100 bar, this case is representative of cryogenic coaxial rocket combustor and has been widely studied [18,21-24].

An injector lip with a height of $h=0.5 \mathrm{~mm}$ separates the two streams of high speed $\mathrm{GH} 2$ in the surroundings and dense LOX in the centre. The computational domain consists of a two dimensional domain of $15 \mathrm{~h} \times 10 \mathrm{~h}$ where the area of interest is limited axially to $10 \mathrm{~h}$ from the splitter face and the remaining region of $5 \mathrm{~h}$ is a sponge layer used to deal with pressure oscillations eventually due to the outlet. A grid convergence study is shown in [20]. Even though, the mesh with 250 grid points along the splitter is chosen in the reference work [20], this present study follows $[16,17]$ by adopting a coarser mesh with a uniform grid spacing of $\Delta \mathrm{x} / \mathrm{h}=\Delta \mathrm{y} / \mathrm{h}=100$. It has been demonstrated to not having noticeable differences with respect to the finer meshes presented in [20]. Similarly to [16], this resolution is applied for the region up to $10 \mathrm{~h}$ in the $\mathrm{x}$-direction and $1.5 \mathrm{~h}$ on both sides of the lip center in the $\mathrm{y}$-direction while the remaining part of the domain is stretched using a factor of 1.02 in the transverse direction. 


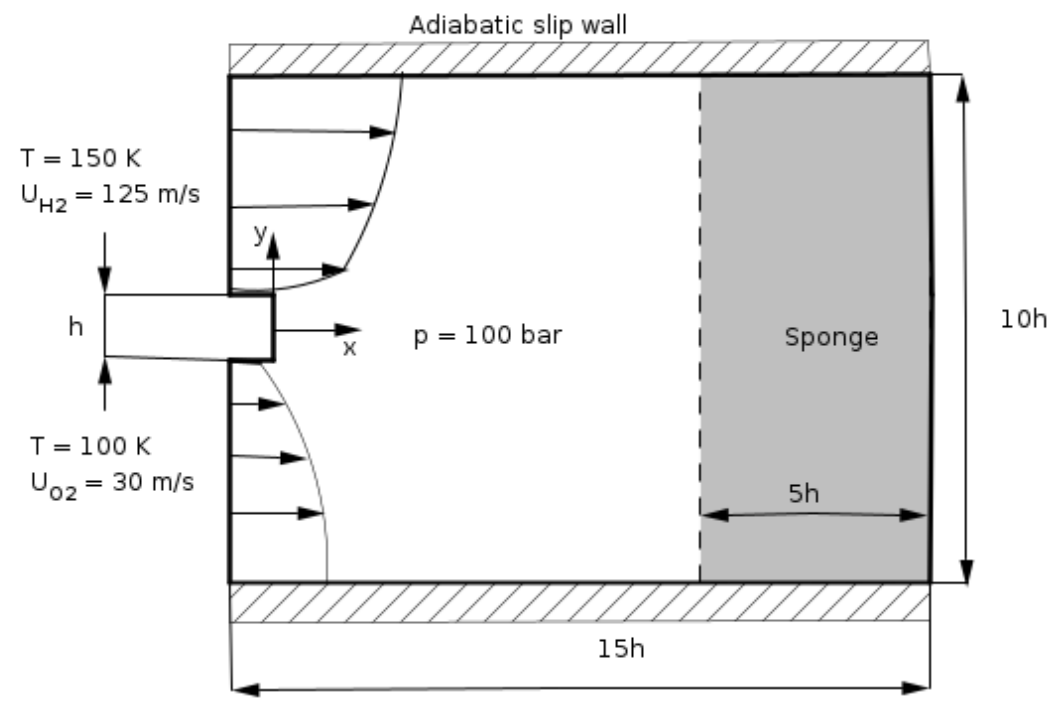

Figure 1. Schematic of the case geometry and boundary conditions.

In addition, the inlet velocities profiles in both streams follow a 1/7 power law. It is noteworthy to mention that the Reynolds numbers are respectively $\operatorname{Re}_{\mathrm{O} 2}=50,000$ and $\mathrm{Re}_{\mathrm{H} 2}=200,000$ based on the splitter height and the injections velocities; while the density ratio is on the order of 80 . An adiabatic no-slip wall is applied at the injector lip whereas the top and the bottom are treated as adiabatic slip walls and a Dirichlet pressure boundary is imposed at the outlet. DNS and LES cases are then considered in the present study.

\section{Model description}

\section{Governing equations}

The governing equations of a fully conservative homogeneous, multicomponent and compressible non reacting two phase flow which are the mass, momentum, energy and species conservation equations, are respectively summarized below:

$$
\begin{aligned}
& \frac{\partial \rho}{\partial t}+\nabla \cdot(\rho \mathbf{U})=0 \\
& \frac{\partial \rho \mathbf{U}}{\partial t}+\nabla \cdot(\rho \mathbf{U} \mathbf{U})=\nabla \cdot(-p \mathbf{I}+\boldsymbol{\tau}) \\
& \frac{\partial\left(\rho h_{T}\right)}{\partial t}+\nabla \cdot\left(\rho h_{T} \mathbf{U}\right)=\frac{\partial p}{\partial t}+\nabla \cdot(\mathbf{U} \cdot \boldsymbol{\tau})-\nabla \cdot \mathbf{q} \\
& \frac{\partial\left(\rho Y_{i}\right)}{\partial t}+\nabla \cdot\left(\rho Y_{i} \mathbf{U}\right)=-\nabla \cdot \mathbf{J}_{i}
\end{aligned}
$$

where $\rho$ is the density, $\mathbf{U}$ is the velocity vector, $p$ is the pressure, $Y_{i}$ refers to the mass fraction of species $i, \mathbf{J}_{i}$ is the species diffusion flux of species $i, \boldsymbol{\tau}$ is the viscous stress tensor, and $\mathbf{q}$ is the heat flux. The energy equation is expressed in terms of total enthalpy with $h_{T}=h+1 / 2 \mathbf{U}^{2}$. The viscous stresses are deduced as for the classical compressible Newtonian fluid model. Contributions of the species diffusion with different enthalpies are neglected when evaluating the heat fluxes using the Fourier's law. Mass diffusion fluxes are modeled using Fick's law. 


\section{Thermophysical models}

To evaluate the thermodynamic fluid properties under supercritical pressure, the Peng-Robinson cubic equation of state (PR-EOS) is used and reads as follows for a mixture $m$

$$
p(v, T)=\frac{R T}{v-b_{m}}-\frac{a_{m}}{v^{2}+2 b_{m} v-b_{m}^{2}}
$$

where $v$ is the molar volume, $b_{m}$ is the effective molecular volume, $a_{m}$ is the attractive force between molecules, $R$ is the gas constant. For multicomponent mixtures, the coefficients $a_{m}$ and $b_{m}$ depend on the critical properties and are calculated using the widely used one-fluid mixture concept with the classical combination rules given by:

$$
\left\{\begin{array}{l}
a_{m}=\sum_{i} \sum_{j} x_{i} x_{j} a_{i j} \\
b_{m}=\sum_{i} x_{i} b_{i} \\
a_{i j}=\left(1-k_{i j}\right) \sqrt{a_{i}} \sqrt{a_{j}},
\end{array}\right.
$$

where $x_{i}$ refers to the molar fraction of the species $i$ and $k_{i j}$ to the binary interaction parameter between the species $i$ and $j$. The above parameters are defined as follows for a species $i$ :

$$
a_{i}=0.455724 \frac{R^{2} T_{c, i}^{2}}{P_{c, i}} \alpha_{i}, b_{i}=0.07780 \frac{R T_{c, i}}{P_{c, i}}, \alpha_{i}=\left[1+c_{i}\left(1-\sqrt{\frac{T}{T_{c, i}}}\right)\right]^{2}
$$

with

$$
c_{i}= \begin{cases}0.37464+1.54226 \omega_{i}-0.26992 \omega_{i}^{2} & \text { if } \omega_{i} \leq 0.5 \\ 0.3796+1.485 \omega_{i}-0.1644 \omega_{i}^{2}+0.01667 \omega_{i}^{3} & \text { otherwise. }\end{cases}
$$

Here and for the remaining part of this work, the subscript "c" refers to the critical condition and $\omega_{i}$ indicates the acentric factor of the species $i$. It should be noted that different mixing rules and combining rules are applied in [19] which are based on the linear blend Kay's rule. The critical properties used within the scope of this current study is summarized in Table 1.

As mentioned previously, with increasing pressure, intermolecular forces are now playing role in the process. Thermodynamic states no longer depend only on temperature, as for the idealized fluids, but also on pressure. The departure formalism are then introduced to determine mixture caloric properties. The latter is expressed as the sum of the ideal gas contribution calculated at the desired temperature and at low reference pressure, and the dense fluid correction which incorporates the pressure effects, also known as departure function. The departure function for the enthalpy $h$ for a given temperature and pressure, for example, can be written as follows:

$$
h(p, T)=h_{0}(p, T)+\int_{p_{0}}^{p}\left[v-T\left(\frac{\partial v}{\partial T}\right)_{p}\right] d p,
$$

where the subscript " 0 ' refers to the ideal reference state (at $p_{0}=1 \mathrm{bar}$ ) and is calculated using the standard 9-coefficients NASA polynomials. On the other hand, the integral part is the departure function which is evaluated from the EOS. Hence, it yields as follows for a mixture $m$ :

$$
h_{m}(p, T)-h_{0, m}(p, T)=p v_{m}-R T+\frac{1}{2 b_{m} \sqrt{2}}\left[a_{m}-T \frac{\partial a_{m}}{\partial T}\right]\left[\frac{v_{m}+b_{m}(1-\sqrt{2})}{v_{m}+b_{m}(1+\sqrt{2})}\right]
$$




\section{Transport Properties}

Chung's correlations with high pressure correction are implemented to evaluate the dynamic viscosity $\mu$ and the thermal conductivity $\kappa$. The approaches for dilute gas derived from The Chapman-Enskog Theory are extended to fluids at high pressure by adding correlated functions of density and temperature.

The mixture dynamic viscosity is then estimated as follows:

$$
\mu_{m}=\mu_{m}^{*} \frac{36.344\left(M_{m} T_{c, m}\right)^{2}}{V_{c, m}{ }^{2 / 3}}
$$

where $M_{m}$ is the mixture molecular weight, $V_{c, m}$ and $T_{c, m}$ refer respectively to critical volume and the critical temperature of the mixture, and also $\mu_{m}^{*}=\frac{T_{m}^{* 1 / 2}}{\Omega_{v, m}}\left(F_{c, m}\left[\left(G_{2}\right)^{1 / 2}+E_{6} y_{m}\right]\right)+\mu^{* *}$, $y_{m}=\frac{\rho V_{c, m}}{6}, T^{*}=1.2593 \frac{T}{T_{c, m}}$, and finally $F_{c, m}=1-0.2756 \omega_{m}+0.059035 \eta_{m}^{4}+c_{m}$.

The parameter $E_{6}$ is among the constant parameters (from $E_{1}$ to $E_{10}$ ) that are linear function of the acentric factors $\omega_{i}$ and functions of constants given in [25]. These parameters are then used to define the remaining terms in the expression of $\mu_{m}^{*}$ which are developed in details in [25]. Furthermore, the mixture properties indicated by the subscript " $m$ " are evaluated following the combination rules detailed in [25]. This counts also among the modifications made to the model introduced in [19] where similar molar weighted averages based mixing rules as for the EOS are used. The thermal conductivity is evaluated following the same principle as for the dynamic viscosity and as given by Chung et al. [25]. Besides, with this parameter $F_{c, m}$, this method gives a good prediction for a wide variety of fluid states accounting for the shape and the polarities of the fluid.

\section{Implementation}

The above models accounting for real fluid thermodynamics are therefore incorporated within a solver named realFluidReactingFoam, based on PIMPLE algorithm, which is a pressurebased segregated approach combining SIMPLE and PISO. To deal with the non-linearity of the equation of state that leads to a rapid and large variation of the density, the pressure equation is therefore adapted following the example of [15], by introducing a linear expression of the density derived through the Taylor expansion. Furthermore, to ensure a strong coupling of the governing equations, the update of the enthalpy and the thermodynamic properties is moved to be after the first momentum predictor step. For the time discretisation, the first order Euler scheme is used and as for both advection and diffusion, second order accurate Gauss limitedlinear schemes are adopted. Further details about the solver can be found in $[19,26]$. It should also be noted that the current approach does not consider phase change; but instead the adiabatic mixing temperature, also called frozen temperature, is calculated.

Table 1. Critical properties of Hydrogen and Oxygen.

\begin{tabular}{c|ccc}
\hline species & $P_{c}[$ bar $]$ & $T_{c}[\mathrm{~K}]$ & $\omega_{i}$ \\
\hline$H_{2}$ & 13 & 157.6 & 0.0025 \\
$O_{2}$ & 50.45 & 33.2 & -0.218
\end{tabular}

\section{Turbulence Model}

For LES simulations, the sgs viscosity $\nu_{t}$ is modeled using the one-equation eddy viscosity model. 
That is, a transport equation is solved for sgs kinetic energy $k_{s g s}$ :

$$
\frac{\partial\left(\rho k_{s g s}\right)}{\partial t}+\frac{\partial\left(\rho \bar{u}_{j} k_{s g s}\right)}{\partial x_{j}}-\frac{\partial}{\partial x_{j}}\left[\rho\left(\nu+\nu_{t}\right) \frac{\partial k_{s g s}}{\partial x_{j}}\right]=-\rho \tau_{i j} \bar{S}_{i j}-C_{\epsilon} \frac{\rho k_{s g s}^{3 / 2}}{\Delta}
$$

where $\tau_{i j}=-2 \nu_{t} \bar{S}_{i j}+2 / 3 k_{s g s} \delta_{i j}$ is the subgrid scale stress tensor and $\bar{S}_{i j}$ refers to the resolved scale strain rate tensor; $\Delta$ denotes the length scale and $C_{\epsilon}$ is a model constant.

The turbulent viscosity is then obtained using $k_{s g s}$ as follows

$$
\nu_{t}=C_{k} k_{s g s}^{1 / 2} \Delta
$$

where $C_{k}$ refers to another model constant.

\section{Results and discussion}

To assess the simulations results, comparison with those from the conservative approach proposed in [20] are presented. To recall, Ruiz et al. [20] propose two solvers which are based on conservative and non-conservative approach. As reported in [20], the steady state is normally reached after $1.25 \mathrm{~ms}$, the equivalent of 10 flow-through times (10 FTT). Figure 2 shows the instantaneous flow fields of axial velocity, transverse velocity, pressure and density. The present numerical solution is able to capture the three large vortical structures in the velocity fields, expected within $x / h=10$, as well as the steep density gradient. Kelvin-Helmholtz mechanisms generate the initial eddies in the hydrogen downstream, at the top corner of the lip. These structures with the interfacial instabilities lead to the larger vortical elements in the oxygen stream. The "comb-like" or "finger-like" structures that have been described in many experimental studies of transcritical flows under LRE relevant conditions [2,5,27], are also observed in the density field (see Figure 2c).



(a)



(c)

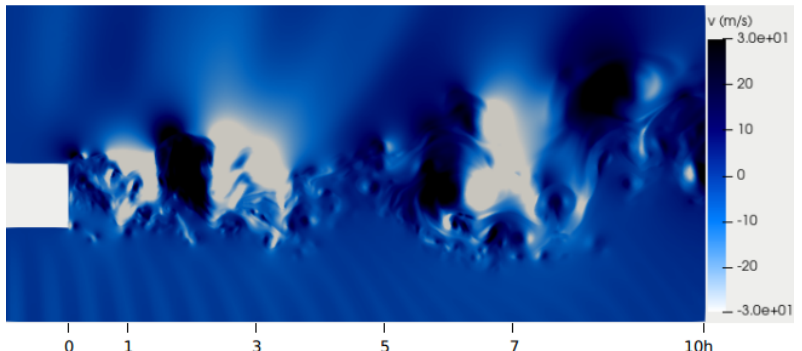

(b)

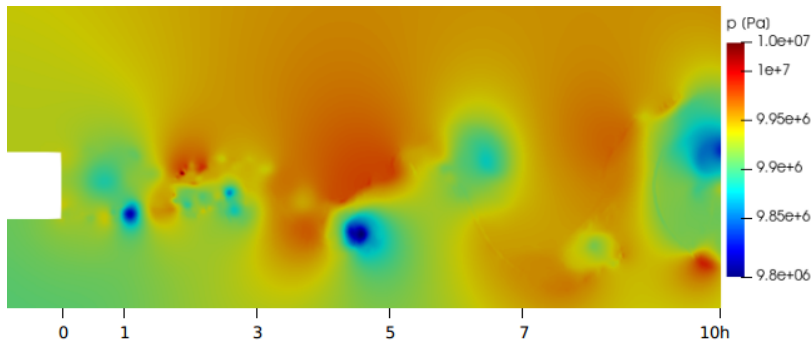

(d)

Figure 2. Instantaneous fields of axial and transverse velocities, density and pressure for the DNS case (from top to bottom and from left to right), at $1.25 \mathrm{~ms}$.

The contour plots of the temperature field and hydrogen mass fraction along with the corresponding scatter plot with respect to the hydrogen mass fraction are shown in Figure 3 . Results are in a good agreement with the simulations presented in [17] for both the energy based (EB) and the enthalpy based (HB) approaches. Figure $3 \mathrm{c}$, in addition to the CFD scatter data, also 
includes phase boundaries from off-line vapor-liquid-equilibrium (VLE) calculations and the adiabatic frozen temperature profile, as references. The comparison clearly shows that, indeed, the oxygen goes through a transcritical thermodynamic process. Additionally, to make further assessments, comparison of statistics collected over time for axial velocity, transverse velocity, pressure, density, temperature, and oxygen mass fraction for both DNS and LES are presented in Figure 4. These mean and root-mean-square statistics (rms) are obtained after 15 FTT and are taken at various axial locations, namely at $\mathrm{x} / \mathrm{h}=1,3,5$ and 7 .

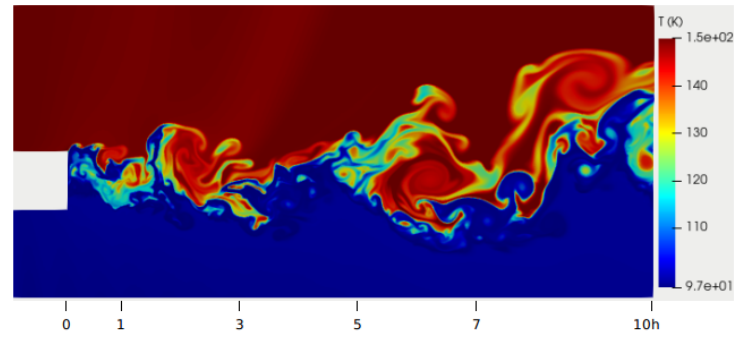

(a)

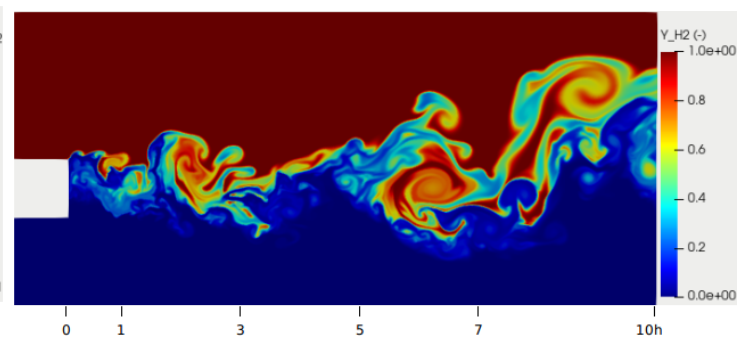

(b)



(c)

Figure 3. Instantaneous fields of hydrogen mass fractions, temperature and scatter plot of temperature versus mass-fraction of hydrogen, at $1.25 \mathrm{~ms}$

Overall, there are good agreements with the general trends of the reference works $[17,20]$ like the asymmetry observed in the rms profiles that broaden towards the hydrogen stream from $x / h=3$ to $x / h=7$. The mean axial velocity profiles shown in Figure $4 a$ are almost identical for the two solvers. However, for the mean transverse velocity profiles, some discrepancies are noted for both DNS and LES cases compared to the results in [20] at $x / h=1$ and 3 , where the transverse velocity is negative due to the recirculation in the wake of the splitter. Nevertheless, the proposed solver is able to capture the upward velocity on the right side at $x / h=5$ as well as the increase of the rms velocity on the oxygen side and its decrease starting at the lip which indicate the growth of the mixing-layer thickness (Figure $4 \mathrm{c}$ and $4 \mathrm{~d}$ ).

Figure $4 \mathrm{e}$ and $4 \mathrm{f}$ show respectively the mean and the rms pressure profiles where DNS and LES results are compared to those of the EB approach in [17]. The mean pressure profile is in good agreement between the different approaches, however the DNS case demonstrates less pressure fluctuations in the oxygen stream. Lacaze et al. attribute the larger pressure oscillations of the EB approach compared to the other cases, to acoustic oscillations in the gradient region. It is worth noting that a longer sponge layer of $15 \mathrm{~h}$ is used in [17] which may also explain the pressure variations between the two solvers. For the density, the temperature and the oxygen mass fraction, both the mean and the rms profiles agree closely with the reference data. For instance, fluctuations up to $y / h=-1.5$ on the oxygen stream and $y / h=3.5$ on the hydrogen side are observed at $\mathrm{x} / \mathrm{h}=7$ in the oxygen $\mathrm{rms}$ profiles for the proposed numerical approach and the reference work (Figure 4l). 


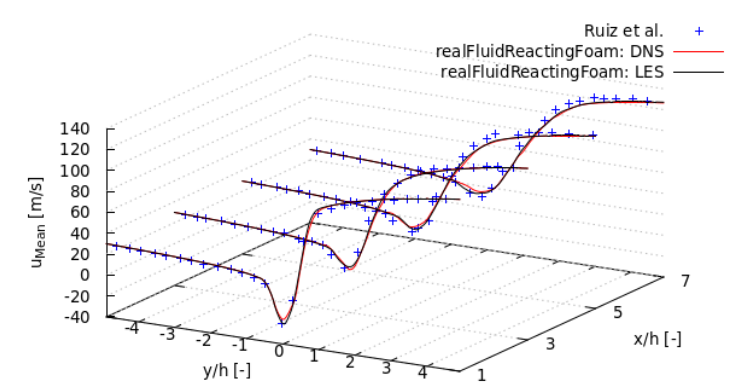

(a)

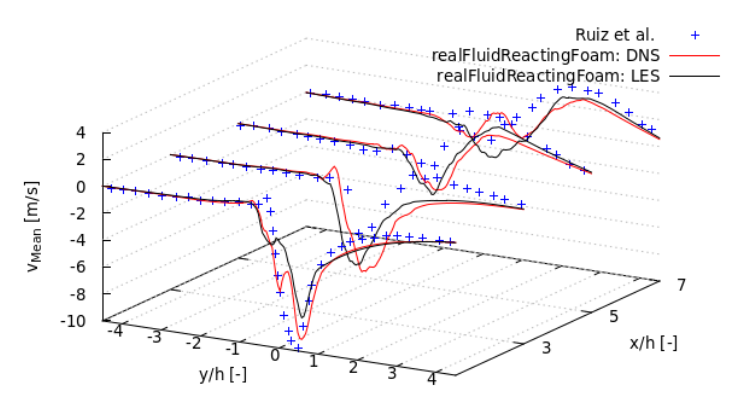

(c)

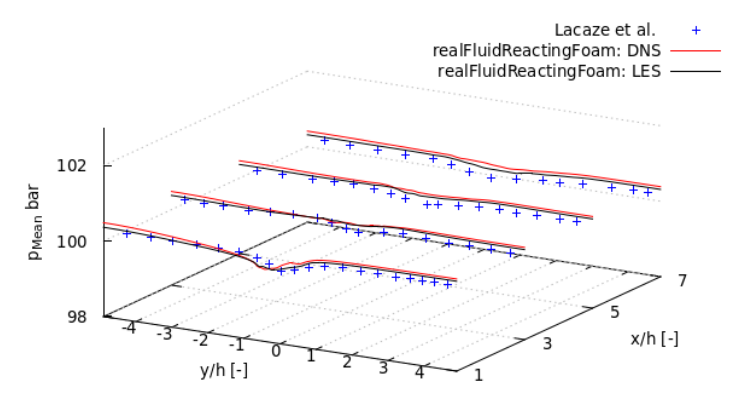

(e)

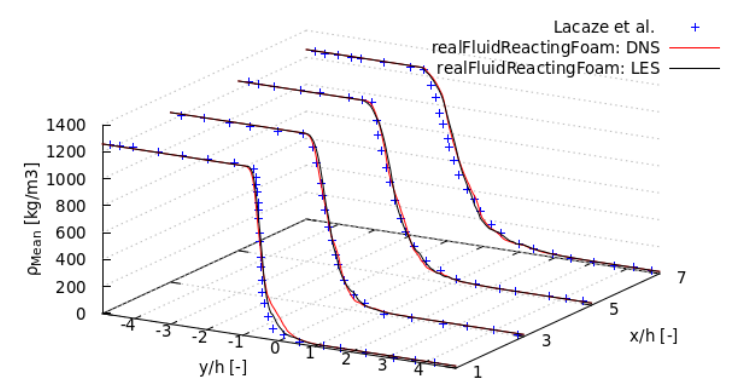

(g)



(b)



(d)

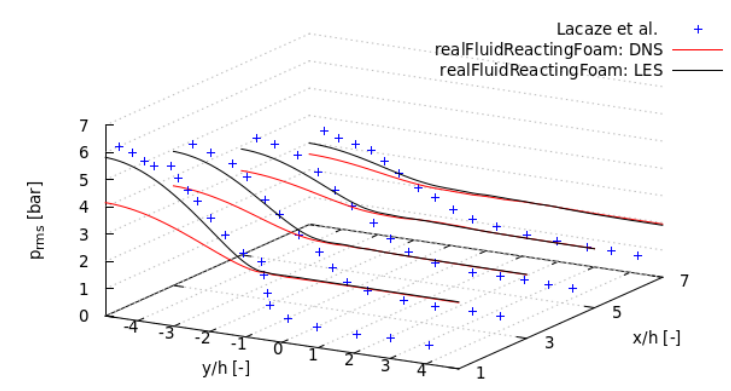

(f)

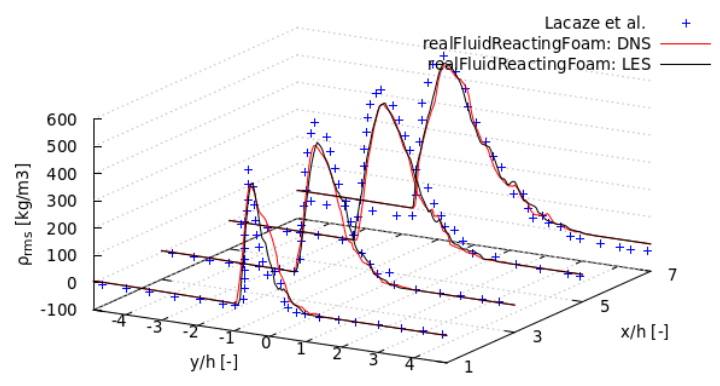

(h)

That is to say, not only the development of the mixing layer towards the hydrogen stream is well captured by the current solver but also the thermophysical characteristics of the flow are adequately described. The LES case appears to not have important influence on the mean profiles for any variable, whereas the rms profiles are in general closer to the reference. 




(i)

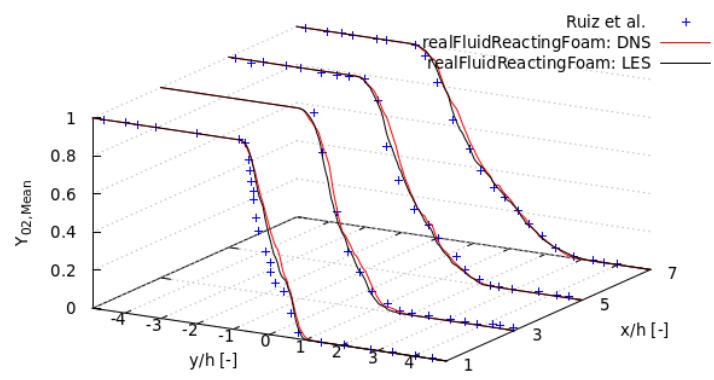

$(\mathrm{k})$

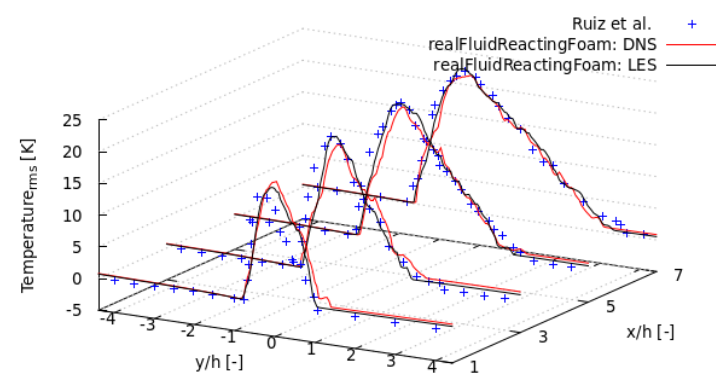

(j)

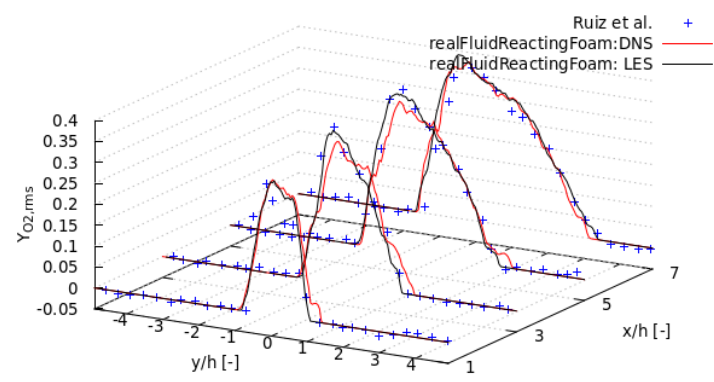

(I)

Figure 4. Transverse cuts of mean (left) and rms (right) axial, transverse velocity, pressure, density, temperature and oxygen mass fraction

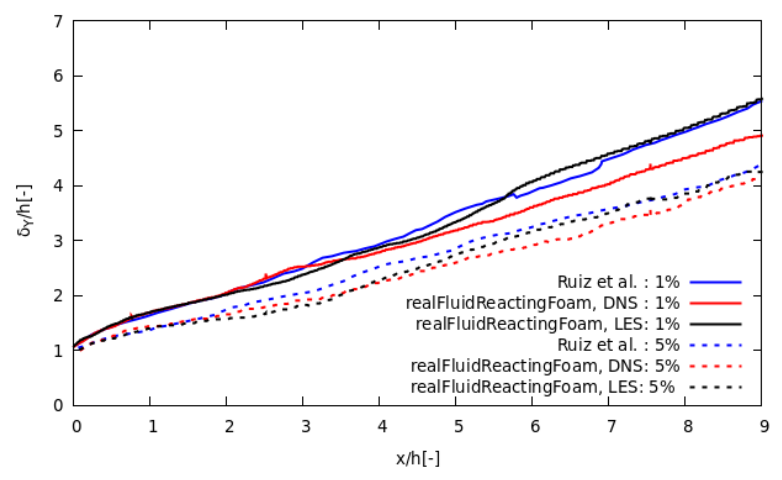

Figure 5. Evolution of the mixing layer thickness in the axial direction

These aforementioned observations are consistent with the evolution of the mixing layer thickness $\delta_{Y}$ in the axial direction presented in Figure 5. The evaluation of the mixing layer thickness at the given location is based on the oxygen mass fraction using $1 \%$ and $5 \%$ thresholds. In other words, the distance between where $\mathrm{Y}_{\mathrm{O} 2}=0.99$ (or $\mathrm{Y}_{\mathrm{O} 2}=0.95$ ) and $\mathrm{Y}_{\mathrm{O} 2}=0.01$ (respectively $\left.\mathrm{Y}_{\mathrm{O} 2}=0.05\right)$ are then evaluated. Lastly, in the following, the impact of the sgs modeling is developed. The evolution of the Reynolds normal (xx, yy) and shear (xy) stresses along the $y$-direction is presented at different axial distances, in Figure 6 . Noticeable differences are obtained between the DNS and the LES cases on the resolved Reynolds stresses for the same computational domain, probably due to insufficient time averaging. The peaks of the Reynolds stresses decay and the profiles broaden toward the positive y side as the flow transition from the wake of the splitter to the mixing layer, hence as the turbulence starts to develop. 


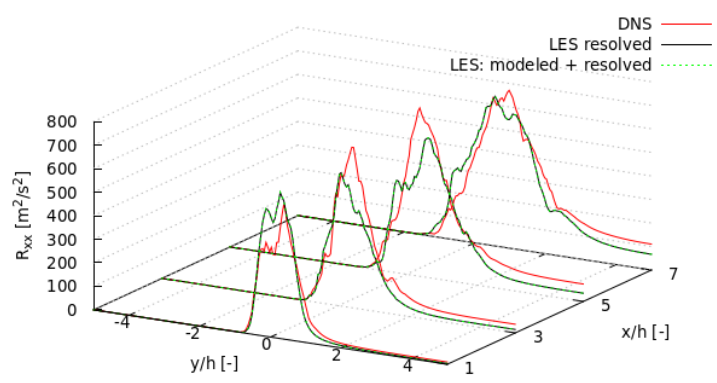

(a)

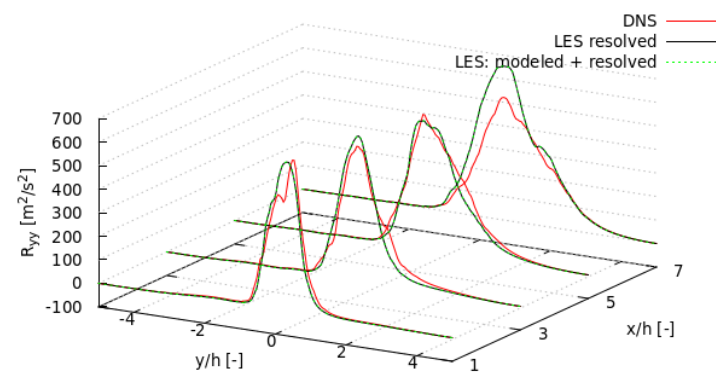

(b)

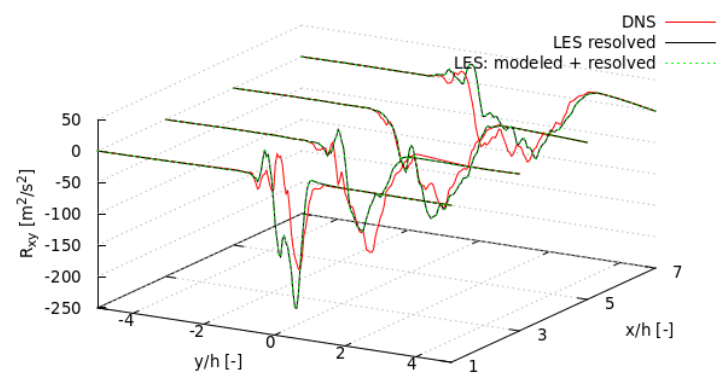

(c)

Figure 6. Influence of sgs modeling on the Reynolds stresses

Figure 7 shows the various contributions to viscosity. Molecular levels, either in DNS or LES, are clearly very similar (Figures $7 \mathrm{a}$ and $7 \mathrm{~b}$ ). In Figure 7c high values of modeled turbulent viscosity in LES are mostly observed in the recirculation zone. This confirms that the mixing formation is mainly triggered by the development of initial large-scale Kelvin-Helmholtz instabilities. The impact of the sgs viscosity is mainly located at the interface between the two fluids, while elsewhere the laminar viscosity obtained through the real-fluid Chung's correlation plays a major role also in the LES.

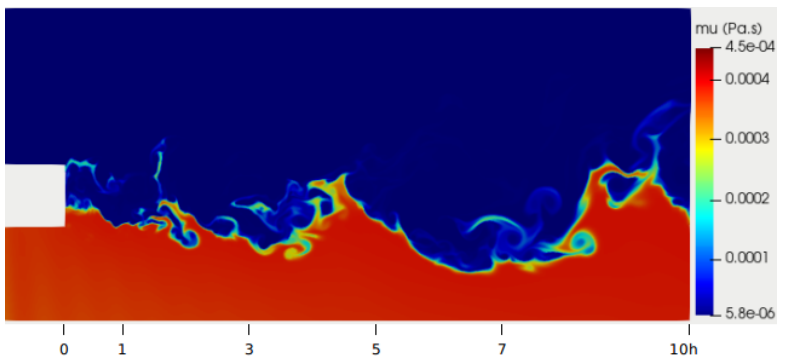

(a) DNS

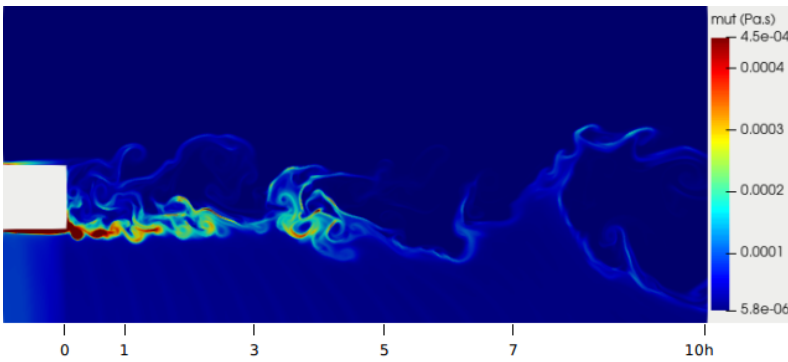

(c) LES

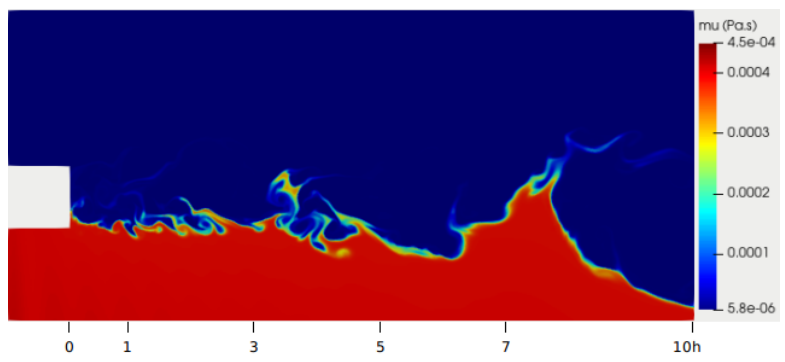

(b) LES



(d) LES

Figure 7. Instantaneous fields of molecular (DNS and LES), turbulent and effective viscosity (from left to right and from top to bottom) 


\section{Conclusions}

The ultimate goal of this work was to contribute to a better understanding of high-Reynoldsnumber supercritical flow with large density gradients. An assessment of the proposed numerical framework capability to handle such simulation conditions with multi-species and real fluid thermodynamics as well as quantitative analysis of the impact of the LES approach have been conducted. Overall, there is a good agreement of the results with the available data in the literature. Besides, no severe pressure or velocity oscillations were recorded. The filtering approach does not play an important role on the mean flows; nevertheless differences are visible in the rms profiles, hence second order moments. This study enables the further development of a real fluid pressure-based multi-species solver as an open-source code.

\section{Acknowledgements}

Authors gratefully acknowledge the support from KAUST, under the CRG grant OSR-2017CRG6-3409.03, and the usage of Shaheen HPC facilities.

\section{References}

[1] Reid, R. C., Prausnitz, J. M., and Poling, B. E., 1987. "The properties of gases and liquids".

[2] Mayer, W., and Tamura, H., 1996. "Propellant injection in a liquid oxygen/gaseous hydrogen rocket engine". Journal of Propulsion and Power, 12(6), pp. 1137-1147.

[3] Habiballah, M., Orain, M., Grisch, F., Vingert, L., and Gicquel, P., 2006. "Experimental studies of high-pressure cryogenic flames on the mascotte facility". Combustion Science and Technology, 178(1-3), pp. 101-128.

[4] Mayer, W., Telaar, J., Branam, R., Schneider, G., and Hussong, J., 2003. "Raman measurements of cryogenic injection at supercritical pressure". Heat and Mass Transfer, 39(8), pp. 709-719.

[5] Oschwald, M., Smith, J., Branam, R., Hussong, J., Schik, A., Chehroudi, B., and Talley, D., 2006. "Injection of fluids into supercritical environments". Combustion science and technology, 178(1-3), pp. 49-100.

[6] Müller, H., Niedermeier, C. A., Matheis, J., Pfitzner, M., and Hickel, S., 2016. "Large-eddy simulation of nitrogen injection at trans-and supercritical conditions". Physics of fluids, 28(1), p. 015102.

[7] Schmitt, T., Selle, L., Ruiz, A., and Cuenot, B., 2010. "Large-eddy simulation of supercritical-pressure round jets". AIAA journal, 48(9), pp. 2133-2144.

[8] Ries, F., Obando, P., Shevchuck, I., Janicka, J., and Sadiki, A., 2017. "Numerical analysis of turbulent flow dynamics and heat transport in a round jet at supercritical conditions". International Journal of Heat and Fluid Flow, 66, pp. 172-184.

[9] Qiu, L., and Reitz, R. D., 2015. "An investigation of thermodynamic states during highpressure fuel injection using equilibrium thermodynamics". International Journal of Multiphase Flow, 72, pp. 24-38.

[10] Bellan, J., 2000. "Supercritical (and subcritical) fluid behavior and modeling: drops, streams, shear and mixing layers, jets and sprays". Progress in energy and combustion science, 26(4-6), pp. 329-366.

[11] Masi, E., Bellan, J., Harstad, K. G., and Okong'o, N. A., 2013. "Multi-species turbulent mixing under supercritical-pressure conditions: modelling, direct numerical simulation and analysis revealing species spinodal decomposition". Journal of Fluid Mechanics, 721, pp. 578-626. 
[12] Foster, J., and Miller, R. S., 2012. "A priori analysis of subgrid mass diffusion vectors in high pressure turbulent hydrogen/oxygen reacting shear layer flames". Physics of Fluids, 24(7), p. 075114.

[13] Matsuyama, S., Shinjo, J., Mizobuchi, Y., and Ogawa, S., 2006. "A numerical investigation on shear coaxial lox/gh2 jet flame at supercritical pressure". In 44th AIAA Aerospace Sciences Meeting and Exhibit, p. 761.

[14] Terashima, H., and Koshi, M., 2012. "Approach for simulating gas-liquid-like flows under supercritical pressures using a high-order central differencing scheme". Journal of Computational Physics, 231(20), pp. 6907-6923.

[15] Jarczyk, M.-M., and Pfitzner, M., 2012. "Large eddy simulation of supercritical nitrogen jets". In 50th AIAA Aerospace Sciences Meeting including the New Horizons Forum and Aerospace Exposition, p. 1270.

[16] Ma, P. C., Lv, Y., and Ihme, M., 2017. "An entropy-stable hybrid scheme for simulations of transcritical real-fluid flows". Journal of Computational Physics, 340, pp. 330-357.

[17] Lacaze, G., Schmitt, T., Ruiz, A., and Oefelein, J. C., 2019. "Comparison of energy-, pressure-and enthalpy-based approaches for modeling supercritical flows". Computers \& Fluids, 181, pp. 35-56.

[18] Oefelein, J. C., and Yang, V., 1998. "Modeling high-pressure mixing and combustion processes in liquid rocket engines". Journal of Propulsion and Power, 14(5), pp. 843-857.

[19] Ningegowda, B. M., Rahantamialisoa, F. N. Z., Pandal, A., Jasak, H., Im, H. G., and Battistoni, M., 2020. "Numerical modeling of transcritical and supercritical fuel injections using a multi-component two-phase flow model". Energies, 13(21), p. 5676.

[20] Ruiz, A. M., Lacaze, G., Oefelein, J. C., Mari, R., Cuenot, B., Selle, L., and Poinsot, T., 2016. "Numerical benchmark for high-reynolds-number supercritical flows with large density gradients". AIAA Journal, 54(5), pp. 1445-1460.

[21] Oefelein, J. C., 2005. "Thermophysical characteristics of shear-coaxial lox-h2 flames at supercritical pressure". Proceedings of the Combustion Institute, 30(2), pp. 2929-2937.

[22] Oefelein, J. C., 2006. "Large eddy simulation of turbulent combustion processes in propulsion and power systems". Progress in Aerospace Sciences, 42(1), pp. 2-37.

[23] Zong, N., and Yang, V., 2006. "Cryogenic fluid jets and mixing layers in transcritical and supercritical environments". Combustion science and technology, 178(1-3), pp. 193-227.

[24] Zong, N., Meng, H., Hsieh, S.-Y., and Yang, V., 2004. "A numerical study of cryogenic fluid injection and mixing under supercritical conditions". Physics of fluids, 16(12), pp. 42484261.

[25] Chung, T. H., Ajlan, M., Lee, L. L., and Starling, K. E., 1988. "Generalized multiparameter correlation for nonpolar and polar fluid transport properties". Industrial \& engineering chemistry research, 27(4), pp. 671-679.

[26] Ningegowda, B. M., Rahantamialisoa, F., Zembi, J., Pandal, A., Im, H. G., and Battistoni, M., 2020. Large eddy simulations of supercritical and transcritical jet flows using real fluid thermophysical properties. Tech. rep., 2020-01-1153 SAE Technical Paper.

[27] Chehroudi, B., 2012. "Recent experimental efforts on high-pressure supercritical injection for liquid rockets and their implications". International Journal of Aerospace Engineering, 2012. 